\title{
TRIVIALITY OF THE HIGHER FORMALITY THEOREM
}

\author{
DAMIEN CALAQUE AND THOMAS WILLWACHER
}

Aвstract. It is noted that the higher version of M. Kontsevich's Formality Theorem is much easier than the original one. Namely, we prove that the higher Hochschild-Kostant-Rosenberg map is already a hoe $_{n+1}$-formality quasi-isomorphism whenever $n \geq 2$.

\section{INTRODUCTION}

Let $A$ be any smooth commutative $\mathbb{K}$-algebra essentially of finite type. We may consider $A$ as an associative $\mathbb{K}$-algebra only, say $A_{1}$. As such we may form its Hochschild cochain complex

$$
C\left(A_{1}\right)=\bigoplus_{k \geq 0} \operatorname{Hom}_{\mathbb{K}}\left(A^{\otimes k}, A\right)[-k]
$$

endowed with the Hochschild differential. The cohomology of $C\left(A_{1}\right)$ is computed by the Hochschild-KostantRosenberg Theorem, which states that the Hochschild-Kostant-Rosenberg (HKR) map

$$
\Phi_{H K R}: S_{A}(\operatorname{Der}(A)[-1]) \longrightarrow C\left(A_{1}\right)
$$

sending a $k$-multiderivation to the obvious map $A^{\otimes k} \rightarrow A$ is a quasi-isomorphism of complexes. Note that $S_{A}(\operatorname{Der}(A)[-1])$ is endowed with the zero differential.

In fact, the degree shifted complexes $S_{A}(\operatorname{Der}(A)[-1])[1]$ and $C\left(A_{1}\right)[1]$ are endowed with differential graded (dg) Lie algebra structures, with the Schouten bracket and the Gerstenhaber bracket, respectively. The central result of deformation quantization is M. Kontsevich's formality Theorem [3], stating that there is an $\infty$-quasiisomorphism of dg Lie algebras

$$
S_{A}(\operatorname{Der}(A)[-1])[1] \longrightarrow C\left(A_{1}\right)[1]
$$

extending the HKR map.

Actually, $S_{A}(\operatorname{Der}(A)[-1])$ also carries the structure of a Gerstenhaber algebra (or, $\mathrm{e}_{2}$ algebra). Kontsevich's result has been strengthened by D. Tamarkin [6], who showed that there also exists an $\infty$-quasi-isomorphism of Ger $_{\infty}$ algebras (or, hoe 2 algebras)

$$
S_{A}(\operatorname{Der}(A)[-1]) \longrightarrow C\left(A_{1}\right)
$$

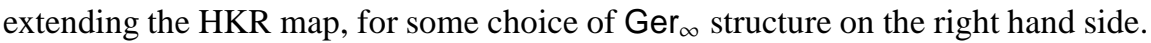

There is a natural generalization of the objects involved to the higher setting. First, we may consider the commutative algebra $A$ as an $\mathrm{e}_{n}=H_{-} .\left(E_{n}\right)$ algebra, say $A_{n}$, with trivial bracket. We assume that $n \geq 2$. We may consider the $\mathrm{e}_{n}$-deformation complex which we also denote by $C\left(A_{n}\right)$. There is a version of the HochschildKostant-Rosenberg Theorem stating that the natural inclusion

$$
\Phi_{H K R}^{n}: S_{A}(\operatorname{Der}(A)[-n]) \rightarrow C\left(A_{n}\right)
$$

is a quasi-isomorphism of complexes.

There is a natural $e_{n+1}$ algebra structure on $S_{A}(\operatorname{Der}(A)[-n])$, with product being the symmetric product and bracket being a degree-shifted version of the Schouten bracket. Similarly, there is an explicit hoe ${ }_{n+1}$ structure on $C\left(A_{n}\right)$, constructed by D. Tamarkin [7]. Here hoe $e_{n+1}=\Omega\left(\mathrm{e}_{n+1}^{\mathrm{i}}\right)$ is the minimal resolution of the operad $\mathrm{e}_{n+1}$, i. e., the cobar construction of the Koszul dual cooperad $\mathrm{e}_{n+1}^{i} \cong \mathrm{e}_{n+1}^{*}\{n+1\}$. The higher formality conjecture states that the (quasi-iso)morphism $\Phi_{H K R}^{n}$ may be extended to an $\infty$-(quasi-iso)morphism of hoe ${ }_{n+1}$ algebras.

The content of the present paper is to point out that this conjecture is somehow trivial.

Theorem 1. For $n \geq 2$ the HKR map $\Phi_{H K R}^{n}: S_{A}(\operatorname{Der}(A)[-n]) \rightarrow C\left(A_{n}\right)$ is already a quasi-isomorphism of hoe h $_{n+1}$ algebras.

D.C. acknowledges the support of the Swiss National Science Foundation (grant 200021_137778). T.W. acknowledges the support of the Swiss National Science Foundation (grants PDAMP2_137151 and 200021_150012). 
This result might be known to the experts, but the authors are unaware of any reference. The proof boils down to a straightforward direct calculation.

Remark 1. As will be clear from the proof the statement of Theorem 1 holds true for $A$ the algebra of smooth functions on a smooth manifold, if one replaces the Hochschild complex by the continuous Hochschild complex, or by the complex of multi-differential operators.

Remark 2. Note that there is a choice in the precise definition of the "Hochschild" complex $C\left(A_{n}\right)$, essentially depending on a choice of cofibrant model for $\mathrm{e}_{n}$. We choose here the minimal model hoe ${ }_{n}$. For some other model $\mathcal{P}$, solving the higher formality conjecture will be "as complicated as" picking a morphism $\mathcal{P} \rightarrow \mathrm{e}_{n}$. The higher formality conjecture for that model can then be recovered by transfer, using Theorem 1 .

Remark 3. Theorem 1 remains valid for any differential graded algebra $A$ as soon as one replaces $\operatorname{Der}(A)$ by its right derived variant $\operatorname{Der}(A)$. Moreover, functoriality of the HKR map allows us to freely sheafify and get in particular that, for a quasi-projective derived scheme $X$ and $n \geq 2$, the HKR map

$$
\Phi_{H K R}^{n}: S_{O_{X}}\left(\mathbb{T}_{X}[-n]\right) \rightarrow C\left(\left(O_{X}\right)_{n}\right)
$$

is a quasi-isomorphism of sheaves of hoe ${ }_{n+1}$ algebras (the only subtlety is to make $C\left(\left(O_{X}\right)_{n}\right)$ into a shear -1$)$. Note that this is slightly different from the main result of [8, Section 5], also called higher formality, where it is proved that the $\mathrm{e}_{n}$ Hochschid complex of $X$ is weakly equivalent to the $E_{n}$ Hochschild complex of $X$ as a Lie L $_{n+1}$ algebra (in our context this is more or less the content of Remark 2, but then the hard part would be to prove that the Lie $\mathrm{L}_{n+1}$ structures on Hochschild complexes appearing in the present paper and the ones appearing in [8] are the same).

Remark 4. Observe that for $n \geq 2$ we have $\mathrm{e}_{n}=$ Pois $_{n}$. All our results and constructions remain valid for every $n \in \mathbb{Z}$ if one uses Pois ${ }_{n}$ in place of $e_{n}$.

Structure of the paper. In section 2 we recall some basic definitions and notation. Section 3 contains a rewording of D. Tamarkin's construction of the hoe ${ }_{n+1}$ algebra structure on $C\left(A_{n}\right)$. The proof of Theorem 1 is a small direct calculation which is presented in section 5 .

Acknowledgements. Damien Calaque thanks Bertrand Toën for explaining him that HKR is true without any assumption in the derived context and for many discussions about the formality for derived schemes and stacks.

\section{NotaTion}

We will work over a ground field $\mathbb{K}$ of characteristic 0 ; all algebraic structures should be understood over $\mathbb{K}$. We will use the language of operads throughout. A good introduction can be found in the textbook [5], from which we freely borrow some terminology.

For a (co)augmented (co)operad $O$ we denote by $O_{\circ}$ the (co)kernel of the (co)augmentation. It is a pseudo(co)operad: i. e. it does not have a (co)unit.

\subsection{Our favorite operads.}

2.1.1. The $\mathrm{e}_{n}$ operad. We will denote by $\mathrm{e}_{n}$ the homology of the topological operad $E_{n}$, for every $n \geq 1$. Note that we work with cohomological gradings (i.e. our differentials have degree +1 ), so that homology sits in non-positive (cohomological) degree.

As an example, $\mathrm{e}_{1}$ is the operad governing non-unital associative algebras. For $n \geq 2$, the operad $\mathrm{e}_{n}$ is isomorphic to an operad obtained by means of a distributive law: $\mathrm{e}_{n} \cong \operatorname{Com}_{\mathrm{Lie}}$, where Lie $_{n}:=\operatorname{Lie}\{n-1\}:=\mathcal{S}^{1-n}$ Lie is a degree shifted variant of the Lie operad. In particular, the space $\mathrm{e}_{n}(N)$ is spanned by formal linear combinations of "Gerstenhaber words", like

$$
\left[X_{1}, X_{2}\right] \cdot X_{4} \cdot\left[X_{3}, X_{5}\right]
$$

in $N$ formal variable $X_{1}, \ldots, X_{N}$, each occurring once. We thus have an obvious map Lie $_{n} \rightarrow \mathrm{e}_{n}$.

\footnotetext{
${ }^{1}$ One shall use the quasi-isomorphic sub-complex of multi-differential operators in $C\left(A_{n}\right)$, which sheafifies well.
} 
2.1.2. The hoe h $_{n}$ operad. The minimal resolution of $\mathrm{e}_{n}$, resp. $\mathrm{Lie}_{n}$, is denoted by hoe ${ }_{n}$, resp. hoLie ${ }_{n}$. In particular hoe h $_{n}=\Omega\left(\mathrm{e}_{n}^{i}\right)$ where $\Omega(\cdot)$ denotes the cobar construction and $\mathrm{e}_{n}^{i} \cong \mathrm{e}_{n}^{*}\{n\}$ is the Koszul dual cooperad of $\mathrm{e}_{n}$. Note that hoe $_{n}$ and hoLie ${ }_{n}$ are dg operads.

One may understand elements of $\mathrm{e}_{n}^{*}(N)$ by linear combinations of "co-Gerstenhaber words", like

$$
\underline{X_{1} X_{2}} \cdot X_{4} \cdot \underline{X_{3} X_{5} X_{6}},
$$

in $N$ formal variable $X_{1}, \ldots, X_{N}$, each occurring once. The underline shall indicate that one equates linear combinations that correspond to (signed) sums of shuffle permutations to zero.

We may also consider the extended $\mathrm{e}_{n}$ operad $\mathrm{ue}_{n}=\mathrm{uCom} \circ \mathrm{Lie}_{n}$, which contains one nullary operation, i.e. $\mathrm{ue}_{n}(0)=\mathrm{uCom}(0)=\mathbb{K}$. It governs unital $\mathrm{e}_{n}$-algebras and can be obtained as the homology of the topological little disks operad, which has a nullary operation acting by deleting disks.

2.1.3. The preLie operad. We will denote by preLie the operad encoding pre-Lie algebras. Following [1], it admits the following combinatorial description. We first introduce the set $\mathcal{T}(I)$ of rooted trees with vertices labelled by a finite set $I$, which is constructed via the following inductive process:

- $\mathcal{T}(\emptyset)$ is empty.

- $\mathcal{T}(\{i\})$ consists of single rooted tree having only one root-vertex labelled by $i$ : $i$

- Let $I$ be a finite set, $i \in I$ and a partition $I_{1} \sqcup \cdots \sqcup I_{k}=I-\{i\}$. Given rooted trees $\mathbf{t}_{\alpha} \in \mathcal{T}\left(I_{\alpha}\right), \alpha=1, \ldots, k$ one can construct a new rooted tree $B_{+}\left(\mathbf{t}_{1}, \ldots, \mathbf{t}_{k}\right) \in \mathcal{T}(I)$ by grafting the root of each $\mathbf{t}_{\alpha}, \alpha=1, \ldots, k$, on a common new root labelled by $i$ :<smiles>[3H]OC</smiles>

Then preLie $(I)$ is the vector space generated by $\mathcal{T}(I)$, and the operadic composition can be defined in the following way: if $J$ is another finite set, $i \in I, \mathbf{t} \in \mathcal{T}(I)$ and $\mathbf{t}^{\prime} \in \mathcal{T}(J)$, then $\mathbf{t} \circ_{i} \mathbf{t}^{\prime}$ is described as a sum over the set of functions $f$ from incoming edges at the vertex $i$ of $\mathbf{t}$ to the vertices of $\mathbf{t}^{\prime}$. For any such $f$, the corresponding term is obtained by removing the vertex $i$ from $\mathbf{t}$, reconnecting the outgoing edge to the root of $\mathbf{t}^{\prime}$ and reconnecting the incoming edges $e$ to the vertex $f(e)$. The root of the result is taken to be the root of $\mathbf{t}$ if this is not $i$, or the root of $\mathbf{t}^{\prime}$ otherwise.

Note that for any operad $O$, the vector spaces $\prod_{n \geq 0} O(n)$ and $\prod_{n \geq 0} O(n)^{S_{n}}$ are naturally preLie algebras.

Recall also that there is a morphism of operads Lie $\rightarrow$ preLie which sends the generator of Lie to (1) - (2) Hence any pre-Lie algebra is also a Lie algebra (obtained by skew-symmetrizing the pre-Lie product).

2.2. The Hochschild complex of a hoe ${ }_{n}$ algebra. For a hoe ${ }_{n}$ algebra $B$, we define the "Hochschild" complex as the degree shifted convolution dg Lie algebra

$$
C(B)=\operatorname{Conv}\left(\mathrm{ue}_{n}^{*}\{n\}, \operatorname{End}_{B}\right)[-n]
$$

where $\operatorname{End}_{B}$ is the endomorphism operad of $B$ and the differential is the Lie bracket with the element of $C(B)$ corresponding to the hoe $_{n}$ structure. In particular, if $B=A_{n}$ is as in the introduction, then

$$
C(B) \cong A \oplus \operatorname{Conv}\left(\mathrm{e}_{n}^{*}\{n\}, \operatorname{End}_{A}\right)[-n] \text {. }
$$

as complexes.

Note that there is a natural inclusion

$$
\Phi_{H K R}^{n}: S_{A}(\operatorname{Der}(A)[-n]) \longrightarrow C\left(A_{n}\right)
$$

whose image consists of the elements in

$$
\operatorname{Conv}\left(u \operatorname{Com}^{*}\{n\}, \operatorname{End}_{A}\right)
$$

that furthermore are (i.e. take values in) derivations in each slot. Analogously to the usual HKR Theorem one may check the following result.

Theorem 2 (Higher Hochschild-Kostant-Rosenberg Theorem). The map $\Phi_{H K R}^{n}$ is a quasi-isomorphism of complexes for each $n \geq 2$.

Sketch of proof. One simply observes that, since the bracket on $A$ is zero, we have

$$
\operatorname{Conv}\left(\text { ue }_{n}^{*}\{n\}, \operatorname{End}_{A}\right)[-n]=S_{A}\left(\operatorname{Conv}\left(\operatorname{Lie}^{*}\{1\}, \operatorname{End}_{A}\right)[-n]\right)=S_{A}(\operatorname{Der}(A)[-n]) .
$$

If $A$ is smooth and essentially of finite type then the canonical map $\operatorname{Der}(A) \rightarrow \operatorname{Der}(A)$ is a quasi-isomorphism. 
3. A version of D. Tamarkin's proof of the higher Deligne conjecture

The goal of this section is to recall D. Tamarkin's proof of the following result.

Theorem 3 (Higher Deligne conjecture, see [7]). The complex $C\left(A_{n}\right)$ carries a natural hoe ( $_{n+1}$ action, given by explicit formulas, for $n \geq 2$.

3.1. Braces for a Hopf cooperad. For any coaugmented cooperad $C$ we may define its bar construction $\Omega(C)$, which is an operad. For example hoe ${ }_{n}:=\Omega\left(e_{n}^{i}\right)$. Here we define a similar construction, the brace construction, which takes a Hopf cooperad $C$ and returns an operad $\mathrm{Br}_{C}$.

3.1.1. $C$-operads. In this paragraph we introduce the notion of a $C$-operad. A $C$-operad is an operad $O$ such that each $O(n)$ carries an $S_{n}$ equivariant right $C(n)$ module structure. We require that furthermore the right module structures are compatible with the operadic compositions, by which we mean that the following diagram commutes:

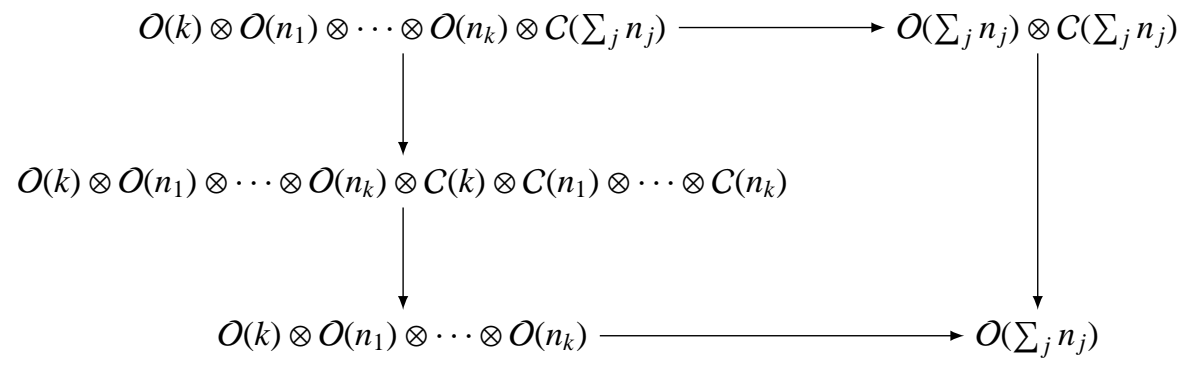

Here the two horizontal arrows are the operadic compositions. The upper left vertical arrow is defined using the cooperad structure on $C$. The remaining two arrows are defined by using the right action of $C(n)$ on $O(n)$. Note that for $C=$ uCom $^{*}$ a $C$-operad is just an ordinary operad.

Example 1. One can check that for an operad $\mathcal{P}$ and a Hopf cooperad $C$, the convolution operad $\operatorname{Hom}(C\{k\}, \mathcal{P})$ is naturally a $C$-operad for any $k$. Here the right action is obtained by composition with the multiplication on $C(n)$ from the right, i. e.,

$$
(f \cdot c)(x)=f(c x)
$$

for $f \in \operatorname{Hom}(C\{k\}, \mathcal{P})(n), c \in C(n)$ and $x \in C\{k\}(n)$.

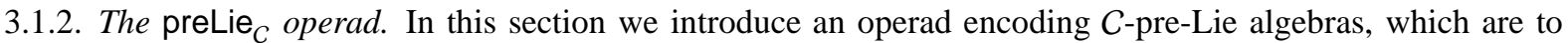
$C$-operads what pre-Lie algebras are to operads. For simplicity, we will assume that the Hopf cooperad $C$ satisfies: $C(0) \cong C(1) \cong \mathbb{K}$. Then one has natural maps

$$
C(j) \rightarrow C(j+k) \otimes \underbrace{C(1) \otimes \cdots C(1)}_{j \times} \otimes \underbrace{C(0) \otimes \cdots C(0)}_{k \times} \cong C(j+k)
$$

where the arrow is a cocomposition and the right hand identification uses the canonical identifications $C(0) \cong$ $C(1) \cong \mathbb{K}$ as algebras.

Example 2. The most interesting example for us is the Hopf cooperad $C=u e_{n}^{*}$, whose $j$-ary cooperations may be interpreted as the cohomology of the configuration space of $j$ points in $\mathbb{R}^{n}$. There are forgetful maps from the configuration space of $k+j$ points to that of $j$ points, and in this case the extension map (1) above is just the pull-back of the forgetful map, forgetting the location of the last $k$ points.

The operad preLie ${ }_{C}$ consists of rooted trees decorated by a Hopf cooperad $C$. Namely, for every finite set $I$,

$$
\operatorname{preLie}_{C}(I):=\bigoplus_{\mathbf{t} \in \mathcal{T}(I)}\left(\bigotimes_{i \in I} C\left(\mathbf{t}_{i}\right)\right),
$$

where $\mathbf{t}_{i}$ is the set/number of incoming edges at the vertex labelled by $i$.

The operadic structure on the underlying trees is the one described in section 2.1.3. Let us now explain what happens to the decoration when doing the partial composition $\circ_{i}$. Borrowing the notation from $\$ 2.1 .3$, for every $f$ we apply a cooperation:

$$
C\left(\mathbf{t}_{i}\right) \longrightarrow \bigotimes_{j \in J} C\left(f^{-1}(j) \cup \mathbf{t}_{j}^{\prime}\right)
$$

\footnotetext{
${ }^{2}$ Note that possible cooperations that we may apply to elements of $C\left(\mathbf{t}_{i}\right)$ are naturally labelled by rooted trees with leaves labelled by $\mathbf{t}_{i}$. The cooperation we apply here is the one labelled by the tree $\mathbf{t}^{\prime}$, with labelled leaves attached according to $f$ and with the labelling of the vertices of $\mathbf{t}^{\prime}$ disregarded.
} 
Then observe that we have natural maps

$$
C\left(f^{-1}(j) \cup \mathbf{t}_{j}^{\prime}\right) \otimes C\left(\mathbf{t}_{j}^{\prime}\right) \rightarrow C\left(f^{-1}(j) \cup \mathbf{t}_{j}^{\prime}\right) \otimes C\left(f^{-1}(j) \cup \mathbf{t}_{j}^{\prime}\right) \rightarrow C\left(f^{-1}(j) \cup \mathbf{t}_{j}^{\prime}\right)=C\left(\left(\mathbf{t} \circ_{i} \mathbf{t}^{\prime}\right)_{j}\right)
$$

where the first map uses the extension map (1) on the second factor and the second map uses the Hopf structure, i. e., it is the multiplication of the algebra $C\left(f^{-1}(j) \cup \mathbf{t}_{j}^{\prime}\right)$.

The definition is made such that preLie ${ }_{C}$ naturally acts on the convolution "algebra"

$$
\mathrm{Conv}_{0}(C\{k\}, \mathcal{P}):=\prod_{n \geq 0} \operatorname{Hom}(C\{k\}, \mathcal{P})(n)^{S_{n}} .
$$

More generally, for any $C$-operad $O, \prod_{n} O(n)^{S_{n}}$ is a preLie ${ }_{C}$ algebra (and we have already seen that $\operatorname{Hom}(C\{k\}, \mathcal{P})$ is a $C$-operad).

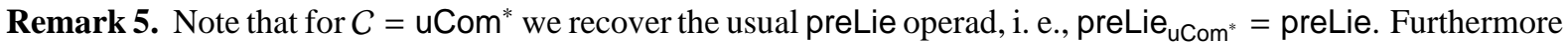
the construction preLie ${ }_{C}$ is functorial in $C$. Hence from the unit map uCom* $\rightarrow C$ we obtain a map of operads preLie $\rightarrow$ preLie $_{C}$ for any Hopf cooperad $C$. In particular, any preLie ${ }_{C}$ algebra is a Lie algebra, and we recover the usual Lie algebra structure on $\operatorname{Conv}_{0}(C\{k\}, \mathcal{P})$.

Next, if we have a morphism $f: \Omega(C\{k\}) \rightarrow \mathcal{P}$ of operads, then it determines a Maurer-Cartan element $\gamma_{f}$ in $\operatorname{Conv}_{0}(C\{k\}, \mathcal{P})$, and a new convolution dg Lie algebra $\operatorname{Conv}_{f}(C\{k\}, \mathcal{P})$ is obtained from the original one one by twisting with $\gamma_{f}$. Note that we may drop the " $f$ " from the notation when there is no ambiguity. In general, the action of the operad preLie ${ }_{C}$ will unfortunately not lift to an action on the twisted convolution Lie algebra $\operatorname{Conv}_{f}(C\{k\}, \mathcal{P})$. However, we now may invoke the formalism of operadic twisting [2]. Given an operad $\mathcal{P}$ together with a map Lie $\rightarrow \mathcal{P}$, operadic twisting produces:

- An operad $T w \mathcal{P}$, the twisted operad.

- Operad maps Lie $\rightarrow T w \mathcal{P} \rightarrow \mathcal{P}$ whose composition is the given map Lie $\rightarrow \mathcal{P}$.

- The operad $T w \mathcal{P}$ has the property that if we are given a $\mathcal{P}$ algebra $A$, together with a Maurer-Cartan element $m$ of the Lie algebra $A$, then the action of the Lie operad on the twisted Lie algebra $A^{m}$ lifts naturally to an action of the operad $T w \mathcal{P}$.

In our case we obtain an operad $\sqrt{3} T w$ preLie $_{C}$, acting naturally on the twisted convolution algebra $\operatorname{Conv}_{f}(C\{k\}, \mathcal{P})$. Concretely the operad $T w$ preLie $_{C}$ is a completed version of the operad generated by preLie ${ }_{C}$ and one formal nullary element. The differential on TwpreLie $_{C}$ is defined so that upon replacing the formal nullary element by the MaurerCartan element $\gamma_{f}$ we obtain an action of $T_{w p r e L i e}$ on $\operatorname{Conv}_{f}(C\{k\}, \mathcal{P})$. The formal nullary element we denote in pictures by coloring the appropriate vertices of the tree black. We call these vertices the internal vertices (as opposed to external ones).

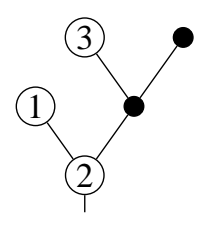

Combinatorially, the differential on $T w$ preLie $_{C}$ splits vertices, either an internal vertex into two internal vertices, or an external vertex into an external and an internal vertex.

We define the brace construction $\operatorname{Br}(C)=T w$ preLie $_{C}$ as a synonym for the twisted pre-Lie operad. By construction $\operatorname{Br}\left(u_{n}^{*}\right)$ acts on the convolution dg Lie algebra

$$
C(B)[n]=\operatorname{Conv}\left(\mathrm{ue}_{n}^{*}\{n\}, \operatorname{End}_{B}\right)
$$

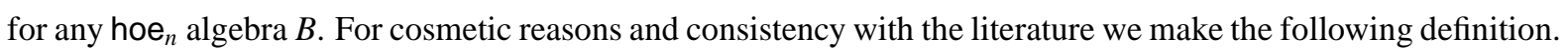

Definition 1. We define the higher braces operad $\mathrm{Br}_{n+1}$ to be the suboperad

$$
\mathrm{Br}_{n+1} \subset \operatorname{Br}\left(\mathrm{ue}_{n}^{*}\right)\{n\}
$$

formed by operations whose underlying trees contain no internal vertices with less than 2 children.

By very definition, the operad $\mathrm{Br}_{n+1}$ acts naturally on the Hochschild complex $C(B)$.

Example 3. The higher braces operad $\mathrm{Br}_{2}$ is just the usual braces operad, which acts on the Hochschild complex of an associative algebra as usual.

\footnotetext{
${ }^{3}$ Which does NOT depend on $f$.
} 
3.2. Tamarkin's morphism. D. Tamarkin proved Theorem 3 by noting that for $n \geq 2$ there is a quite simple but very remarkable explicit map

$$
T: \text { hoe }_{n+1} \rightarrow \mathrm{Br}_{n+1} \text {. }
$$

It is defined on generators by the following prescription:

- Generators of the form $\underline{X_{1} \cdots X_{k}} \in e_{n+1}^{i}(k)$ are mapped to a corolla of the form

(2)

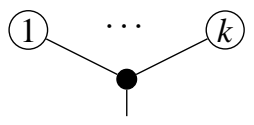

decorated by $X_{1} \cdots X_{k} \in e_{n}^{i}(k)$

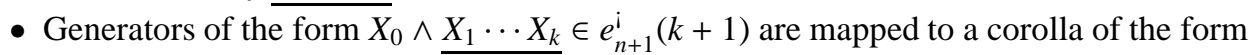

(3)<smiles>[O][O]</smiles>

decorated by $X_{1} \cdots X_{k} \in e_{n}^{i}(k)$. In the special case $k=1$ one takes the (anti-)symmetric combination of the two possible choices.

- All other generators are mapped to zero.

This prescription indeed gives a morphism hoe ${ }_{n+1} \rightarrow \mathrm{Br}_{n+1}$ of the underlying graded operads (because, as such, hoe $_{n+1}$ is free). This leaves us with the task of verifying that the map $T$ : hoe ${ }_{n+1} \rightarrow \mathrm{Br}_{n+1}$ commutes with the differentials. It suffices to check this on the generators. Furthermore it suffices to check the statement on generators of one of the forms

$$
\underline{X_{1} \cdots X_{k}} \quad \underline{X_{1} \cdots X_{k}} \wedge \underline{X_{k+1} \cdots X_{k+l}} \quad X_{1} \wedge \underline{X_{2} \cdots X_{k+1}} \wedge \underline{X_{k+2} \cdots X_{k+l+1}}
$$

since in all other cases the differential of the generator and the generator itself are mapped to zero, so that the map $T$ trivially commutes with the differentials. One has to check each of the three types of generators above in turn. The calculation is a bit lengthy, due to several special cases that need to be considered. Since the construction of $T$ is essentially the result of D. Tamarkin [7] we will only show how to handle a few cases in Appendix $₫$ as an illustration.

\section{4. $\mathrm{Br}_{n}$ IS AN $E_{n}$ OPERAD}

Theorem 4. The above map $T:$ hoe $_{n+1} \rightarrow \mathrm{Br}_{n+1}$ is a quasi-isomorphism of operads for all $n=2,3,4, \ldots$, so in particular $H\left(\mathrm{Br}_{n+1}\right) \cong \mathrm{e}_{n+1}$.

In the case $n=1$ it is still true that there is a quasi-isomorphism hoe $\mathrm{B}_{2} \rightarrow \mathrm{Br}_{2}$, but this morphism is much more complicated to construct than the Tamarkin quasi-isomorphism $T$ we described above. It can be obtained by combining a quasi-isomorphism from $\mathrm{Br}_{2}$ to the chains of the little disks operad [4] with a choice of formality morphism of the little disks operad.

Theorem 4 is not used in this note, so we only sketch the proof.

Sketch of proof. First one checks that $H\left(\mathrm{Br}_{n+1}\right) \cong \mathrm{e}_{n+1}$. The proof of this statement follows along the lines of the proof of the $n=1$ case in [2, Appendix C]. The only point where the proof in loc. cit. has to be adapted is that in [2, Appendix C.2] one has to compute the Hochschild cohomology of a free $\mathrm{e}_{n+1}$ algebra, considered as an $\mathrm{e}_{n}$ algebra, instead of computing the Hochschild cohomology of a free $e_{2}$ algebra, considered as an $e_{1}$ algebra. The answer is provided by the higher Hochschild-Kostant-Rosenberg Theorem, i. e. Theorem 2 above, instead of the usual one.

Once one knows that $H\left(\mathrm{Br}_{n+1}\right) \cong \mathrm{e}_{n+1}$, the statement of the Theorem is shown by checking that the induced map in cohomology

$$
\mathrm{e}_{n+1} \cong H\left(\mathrm{hoe}_{n+1}\right) \rightarrow H\left(\mathrm{Br}_{n+1}\right) \cong \mathrm{e}_{n+1}
$$

is the identity, which amounts to checking that it is the identity on the two generators.

The brace construction $\mathrm{Br}_{n+1}$ is intuitively similar to taking a product of $\Omega\left(\mathrm{e}_{n}^{i}\right)$ with an $E_{1}$ operad. So the above Theorem shall be understood as a version of the statement that the product of an $E_{1}$ operad with an $E_{n}$ operad is an $E_{n+1}$ operad. 


\section{Proof of Theorem 1}

One needs to verify that the action of all generators of hoe ${ }_{n+1}$ commutes with the map. On the left hand side all actions are zero except for that of the generators $X_{1} \wedge X_{2}$ (i.e., the Lie bracket) and of $X_{1} X_{2}$ (i.e., the product). The fact that the HKR morphism respects the Lie bracket is a simple verification. The fact that the product is preserved is obvious. Hence it suffices to check that the action of the generators $X_{1} \cdots X_{k}(k \geq 3)$ and $X_{0} \wedge X_{1} \cdots X_{k}(k \geq 2)$ on the image of the HKR map is trivial. The generators $X_{1} \cdots X_{k}$ act using the corresponding components of the hoe $_{n}$ structure on $A_{n}$, which vanish. Hence they act trivially (as long as $k \geq 3$ ). Note also that the image of the HKR map has only "hoLie ${ }_{n}$ components", i.e., the corresponding maps $\mathrm{e}_{n}^{i}(N) \rightarrow \operatorname{End}(V)(N)$ factor through $\mathrm{e}_{n}^{i}(N) \rightarrow \operatorname{Lie}_{n}^{i}(N)$. However, the prescription for the action of the component $X_{0} \wedge X_{1} \cdots X_{k}$ advises us to evaluate the arguments on components $X_{1} \cdots X_{k}$, which are sent to zero under the projection $\mathrm{e}_{n}^{i}(k) \rightarrow \operatorname{Lie}_{n}^{i}(k)$. Hence the action of the components $X_{0} \wedge \overline{X_{1} \cdots X_{k}}$ vanishes (as long as $k \geq 2$ ) on the image of the HKR map.

\section{ApPEndix A. The Map $T$ COMMUTES With THE DIFFERENTIALS}

A.1. The generator $X_{1} \cdots X_{k}$. In this case the differential of the generator consists of

$$
\sum_{j, r} \pm \underline{X_{1} \ldots X_{j-1} * X_{j+r+1} \ldots X_{k}}{ }^{\circ} * \underline{X_{j} \ldots X_{j+r}}
$$

where the notation $A \circ_{*} B$ shall mean the operadic composition in hoe ${ }_{n+1}$ of the operations $A$ and $B$ in hoe ${ }_{n+1}$, with $B$ being "inserted in the slot" of $A$ labelled by $*$. The map $T$ sends the above to

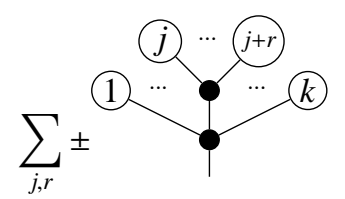

This is precisely the differential of the tree (2), which is the image of $\underline{X_{1} \cdots X_{k}}$ by $T$. Decorations are obvious.

A.2. The generator $X_{0} \wedge X_{1} \cdots X_{k}$. In this case the differential of the generator consists of the following types of (5)

$$
\begin{aligned}
\sum_{j} \sum_{r \geq 1} \pm X_{0} \wedge \underline{X_{1} \cdots X_{j-1} * X_{j+r+1} \cdots X_{k}} & \circ_{*} \underline{X_{j} \cdots X_{j+r}}+\sum_{j} \sum_{r \geq 1} \underline{ \pm X_{1} \cdots X_{j-1} * X_{j+r+1} \cdots X_{k} \circ_{*}}\left(X_{0} \wedge \underline{X_{j} \cdots X_{j+r}}\right) \\
& \pm\left(X_{0} \wedge *\right) \circ_{*} \underline{X_{1} \cdots X_{k}}+\sum_{j} \pm \underline{X_{1} \cdots X_{j-1} * X_{j+2} \cdots X_{k}} \circ_{*}\left(X_{0} \wedge X_{j}\right)
\end{aligned}
$$

This is mapped under $T$ to a linear combination of trees of the following form

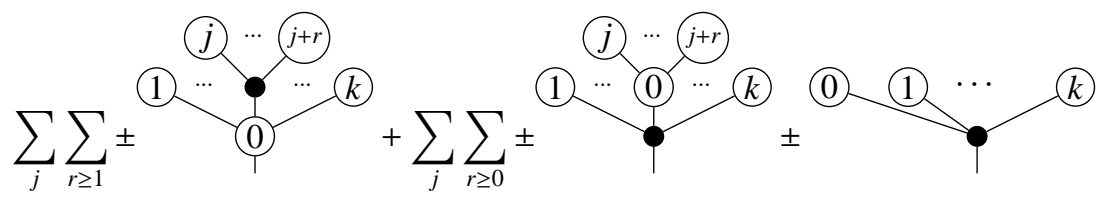

Here all corollas are decorated by the top degree elements of $u e_{n}^{*}$, except for the last tree, where the decoration is by the element $X_{0} \wedge X_{1} \cdots X_{k}$.

One checks that this linear combination of trees is exactly the differential of (3), which is the image of the generator we considered by $T$. Note also that the trees of the form

(0)

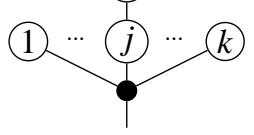

occur twice, with the two contributions from the third and fourth term of (5) cancelling each other.

\section{REFERENCES}

[1] Frédéric Chapoton and Muriel Livernet, Pre-Lie algebras and the rooted trees operad. Int. Math. Res. Not. 2001 (2001), no. 8, $395-408$.

[2] Vasily Dolgushev and Thomas Willwacher, Operadic twisting - with an application to Deligne's conjecture, preprint arXiv: 1207.2180 (2012).

[3] Maxim Kontsevich, Deformation quantization of Poisson manifolds, Lett. Math. Phys. 66 (2003), no. 3, $157-216$.

[4] Maxim Kontsevich and Yan Soibelman, Deformations of algebras over operads and the Deligne conjecture, Conférence Moshé Flato 1999, Vol. I (Dijon), Math. Phys. Stud.21, Kluwer Acad. Publ., (2000), 255-307. 
[5] Jean-Louis Loday and Bruno Vallette, Algebraic Operads, Grundlehren der mathematischen Wissenschaften Vol. 346, 634 p., Springer-Verlag, Berlin, 2012.

[6] Dmitry Tamarkin, Another proof of M. Kontsevich formality theorem, preprint arXiv:math/9803025(1998).

[7] Dmitry Tamarkin, Deformation complex of a d-algebra is a (d+1)-algebra, preprint arXiv:math/0010072 (2000).

[8] Bertrand Toën, Operations on derived moduli spaces of branes, preprint arXiv:1307.0405 (2013).

I3M, Université Montpellier 2, Case courrier 051, 34095 MontPellier cedex 5, France

E-mail address: damien.calaque@univ-montp2.fr

Department of Mathematics, University of Zurich, Winterthurerstrasse 190, 8057 Zurich, Switzerland

E-mail address: t.willwacher@gmail.com 